Article

\title{
Preparation and Characterization of New Nano-Composite Scaffolds Loaded With Vascular Stents
}

\author{
Hongzhen $\mathrm{Xu}{ }^{1}$, Jiansheng Su ${ }^{1} *$, Jun Sun ${ }^{1}$ and Tianbin Ren ${ }^{2}$
}

1 Institute of Prosthodontics, School of Stomatology, Tongji University, 399 Yanchang Road, Shanghai 200092, China; E-Mails: xuhongzhen0531@163.com (H.X.); cipher44444444@hotmail.com (J.S.)

2 Institute of Nano- and Bio-Polymeric Materials, School of Materials Science and Engineering, Tongji University, 1239 Siping Road, Shanghai 200092, China; E-Mail: rtb002@163.com

* Author to whom correspondence should be addressed; E-Mail: ts1967118@ yahoo.com.cn; Tel.: +86-21-6631-1629; Fax: +86-21-6652-4025.

Received: 1 December 2011; in revised form: 4 January 2012 / Accepted: 20 February 2012 / Published: 12 March 2012

\begin{abstract}
In this study, vascular stents were fabricated from poly (lactide- $\varepsilon$ caprolactone)/collagen/nano-hydroxyapatite (PLCL/Col/nHA) by electrospinning, and the surface morphology and breaking strength were observed or measured through scanning electron microscopy and tensile tests. The anti-clotting properties of stents were evaluated for anticoagulation surfaces modified by the electrostatic layer-by-layer self-assembly technique. In addition, nano-composite scaffolds of poly (lactic-co-glycolic acid)/polycaprolactone/nano-hydroxyapatite (PLGA/PCL/nHA) loaded with the vascular stents were prepared by thermoforming-particle leaching and their basic performance and osteogenesis were tested in vitro and in vivo. The results show that the PLCL/Col/nHA stents and PLGA/PCL/nHA nano-composite scaffolds had good surface structures, mechanical properties, biocompatibility and could guide bone regeneration. These may provide a new way to build vascularized-tissue engineered bone to repair large bone defects in bone tissue engineering.
\end{abstract}

Keywords: nano-composite scaffold; tissue engineering; mechanical property; electrospinning 


\section{Introduction}

Bone tissue engineering has provided a new way to repair, restore or regenerate large bone defects impaired by disease, injury, or age [1]. Constructs are typically created by seeding a scaffold with cells in vitro. Scaffolds are an important component in bone tissue engineering and these should be three-dimensional, with a highly porous network of interconnected pores that can promote cell growth, adhesion, proliferation and differentiation by facilitating the flow and transport of nutrients and metabolic waste. In addition, they should have good biocompatibility and biodegradability to ensure they are non-toxic, non-allergic and cause no other adverse reactions to cells and the body and promote the formation of new tissues matched to the degradation rate of stent materials. Furthermore, they should have good biological activity to improve cell viability and promote tissue regeneration. Finally, good mechanical properties are also essential requirements of an ideal scaffold in bone tissue engineering [2-5]. Currently, vascularization has been the greatest problem in the successful repair of large bone defects by bone tissue engineering [1,6]. Therefore, the construction and mechanical, biological and anti-clotting properties of scaffolds are of crucial importance.

The normal vascular anatomy, in addition to capillaries and lymphatic capillaries, includes an outer membrane, a membrane and intimal layers. Endometrial lining is constituted by a single layer of epithelial cells in the basement membrane, and presents a smooth surface, rich in various types of collagen and elastin. The membrane is the thickest layer, with good mechanical properties, composed of smooth muscle cells arranged in layers of interaction. The adventitia is composed of loose connective tissue, which mainly contains fibroblast and type I collagen [7]. Ideal vascular stents should have the following characteristics: (1) they simulate the structure of normal vascular anatomy; (2) they have good biocompatibility and biological characteristics; (3) they have sufficient mechanical properties and compliance [8-10].

Currently, electrospinning has been the main method for tissue engineering of stents in many nano-fiber preparation technologies [11,12]. It has the following major advantages: (1) electrospun fiber stents have good biocompatibility; (2) precursor solutions of electrospinning are diverse; (3) the electrospinning process is highly controllable [13]. Nano-fibrous tissue engineered blood vessel stents have special advantages, as they are not only able to simulate the anatomy of the normal vascular tissue, but can mimic the composition and structure of the extracellular matrix to provide an ideal growing environment for cells, which are important prerequisites for the regeneration and reconstruction of blood vessels. In previous studies, tissue engineering of vascular stents could not fully be taken advantage of because implanting them into the body was prone to rapid thrombopoiesis, which is one of the limiting factors in clinical applications. To overcome this shortcoming, various methods for surface modification and functional modification on the stent surface exist that can improve performance by loading the surface with anti-clotting substances [14,15]. Layer-by-layer assembly has been one of the common means of modifying the stent surface in tissue engineering, which is not only suitable for materials with a variety of complex structures but also can fix a variety of biological molecules on the surface [16].

Collagen is the most abundant protein in the human body and a key element of the extracellular matrix (ECM) which imparts structural integrity and tensile strength to tissues. Recently, it has been used in a lot of tissue engineering applications as its predominance in the ECM, non-immunogenicity 
and available methods of isolation from a variety of sources. In addition, collagen fibers also possess some unique structural properties important for tissue engineering: they transmit forces, dissipate energy, prevent premature mechanical failure and provide biological signals to adjacent cells that regulate functional responses [17]. Collagen is resorbable, has high hydrophilicity, low antigenicity, very good biocompatibility and can promote tissue regeneration [18]. As it has many advantages, it has been one of the most ideal biopolymers available for tissue engineering applications.

Biodegradable polyesters, such as polylactic acid (PLA), polyglycolic acid (PGA) and their copolymers poly (lactic-co-glycolic acid) (PLGA), have good biocompatibility and biodegradation and are approved as a class of biological materials by the United States Food and Drug Administration (FDA). These show good mechanical strength, elastic modulus and can be thermally formed, so meet the basic requirements of carrier materials in bone and cartilage tissue engineering [19-21]. The mechanical properties and degradation rate of PLGA/PCL composite scaffolds can be improved by adjusting the molecular weight, structure and composition to meet different clinical requirements, and their ultimate degradation products are $\mathrm{CO}_{2}$ and $\mathrm{H}_{2} \mathrm{O}$. Intermediate products of lactic acid are also the body's normal sugar, so PLGA/PCL scaffold degradation in vivo will not have adverse effects on the organism [19,22,23]. In addition, excellent performances of composite materials have been widely reported in recent years [3,24]. The introduction of inorganic materials such as hydroxyapatite, calcium silicate not only enhance the mechanical strength of scaffold materials but can neutralize the acid produced by polymer degradation to maintain a stable $\mathrm{pH}$ value $[25,26]$. Synthetic hydroxyapatite, a type of inorganic material, has been extensively studied and applied, and is similar to natural bone. It has good biocompatibility, bioactivity and bone leading properties, and can guide new bone regeneration from the host bone along implant interfaces or internal implants to form a good bone combination [27-30].

In this study, electrospinning technology was applied to prepare small, biomimetic vascular stents of poly (lactide- $\varepsilon$-caprolactone)/collagen/nano-hydroxyapatite (PLCL/Col/nHA), and a modified layer of anti-coagulant which was built on the surface to improve the anti-clotting properties through layer-by-layer assembly technology. New nano-composite scaffolds loaded with vascular stents prepared by tissue engineering technology were used as substitutes for bone matrices, with good biocompatibility and mechanical properties.

\section{Results and Discussion}

\subsection{Construction and Properties of Vascular Stents}

The surface morphology of the vascular stents was observed by scanning electron microscopy (SEM) (Figure 1), exhibiting a highly uniform thickness and ordered arrangement of nano-fibers. Each group of components and concentration is shown in Table 1. The concentration of solution greatly affected the morphology and diameter of electrospun fibers and had to exceed a critical value of electrospinning to carry out the process of electrostatic spinning smoothly. When the solution is diluted, it cannot form a stable fluid and will directly contract under the effects of surface tension and form liquid beads or silk. Mutual entanglement of polymer chains with increasing concentration prevents the deformation of small streams, forming uniform fibers, but if the concentration continues 
to increase, the solution will be gelatinous and not conducive to formation of neat fibers [31]. In this study, the viscosity of the spinning solution increased significantly with increasing PLCL concentration. nHA had less influence on the fiber diameter than collagen at low content. $0.3 \%$ collagen could make the stents exhibit better hydrophilicity and biocompatibility compared with $0.2 \%$, $0.4 \%$ and $0.5 \%$, which had been studied and proved by comparative pre-studies.

Figure 1. Scanning Electron Microscopy (SEM) images of different vascular stents: (a) poly (lactide-e-caprolactone) (PLCL) group; (b) PLCL/nano-hydroxyapatite (nHA) group; (c) PLCL/collagen (Col)/nHA I group; (d) PLCL/Col/nHA II group.

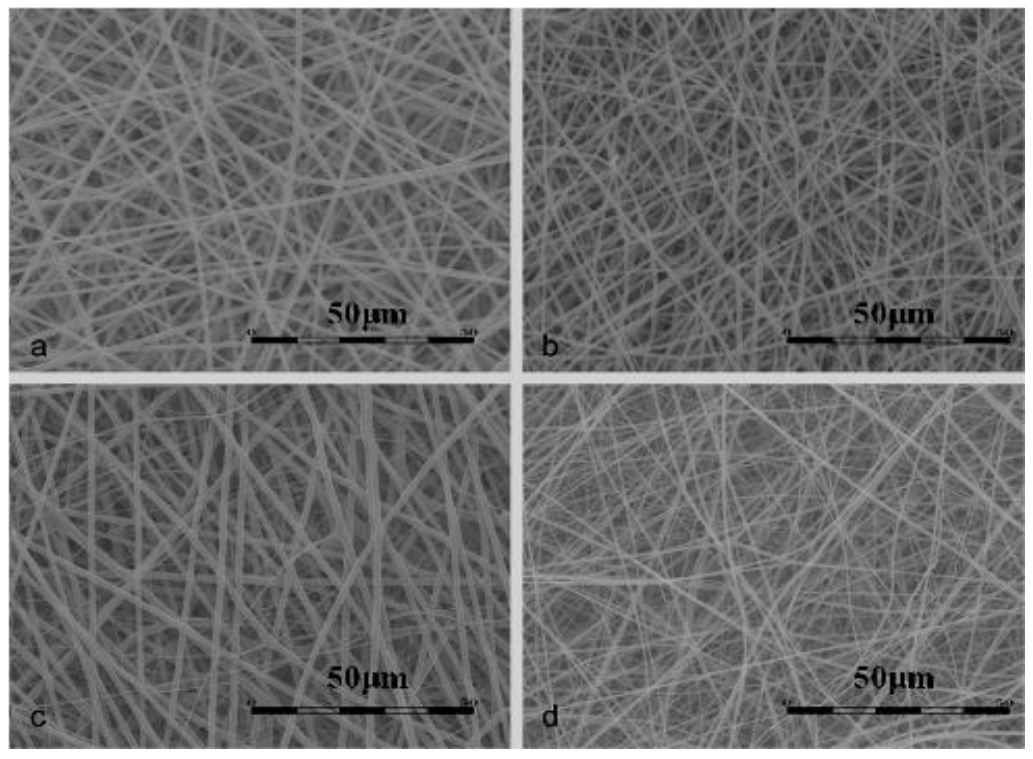

Table 1. The components of each group.

\begin{tabular}{cc}
\hline Group & Components \\
\hline PLCL & PLCL, $10 \%(\mathrm{~g} / \mathrm{mL})$ \\
PLCL/nHA & PLCL, $10 \%(\mathrm{~g} / \mathrm{mL})+\mathrm{nHA}, 0.03 \%(\mathrm{~g} / \mathrm{mL})$ \\
PLCL/Col/nHA I & PLCL, $10 \%(\mathrm{~g} / \mathrm{mL})+\mathrm{Col}, 0.3 \%(\mathrm{~g} / \mathrm{mL})+\mathrm{nHA}, 0.03 \%(\mathrm{~g} / \mathrm{mL})$ \\
PLCL/Col/nHA II & PLCL $+\mathrm{Col}+\mathrm{nHA}, 0.07 \%(\mathrm{~g} / \mathrm{mL})$, PLCL/Col $=1: 2(\mathrm{~g} / \mathrm{g})$ \\
\hline
\end{tabular}

Compared with pure PLCL, the tensile strength of stents was improved slightly with the joining of nHA $(p>0.05)$ but it was significantly reduced through addition of collagen $(p<0.05)$ (Figure 2A). The break elongation ratio showed little change in PLCL/nHA group and PLCL/Col/nHA I group compared with pure PLCL group $(p>0.05)$. When the collagen content of scaffolds was higher than the PLCL content, both the tensile strength and breaking elongation ratio were greatly reduced $(p<0.01)$ (Figure 2B).

In this experiment, the clotting time of the pure PLCL fiber membrane was about $350 \mathrm{~s}$, while the PLCL/Col/nHA II fiber membrane modified through the electrostatic self-assembly technology showed a clotting time far greater than $1800 \mathrm{~s}$ (Figure 3) (all $p$ values compared with control group are less than 0.05). Regardless of whether the outermost modified layers were chitosan (CTS) or heparin sodium (HS), all showed good anticoagulant properties, which may have resulted from the existence of a certain amount of HS on the fiber membrane surfaces, interspersed with the outermost layer. HS has 
good anticoagulant performance so membranes showed a corresponding improvement in performance with increasing HS.

Figure 2. The mechanical properties of different vascular stents evaluated through tensile strength (A) and elongation to break ratio (B).

(A)

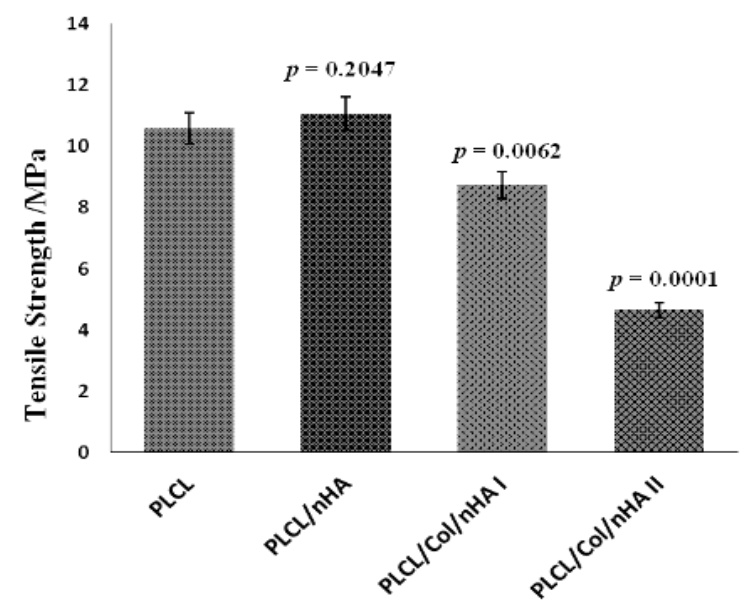

(B)

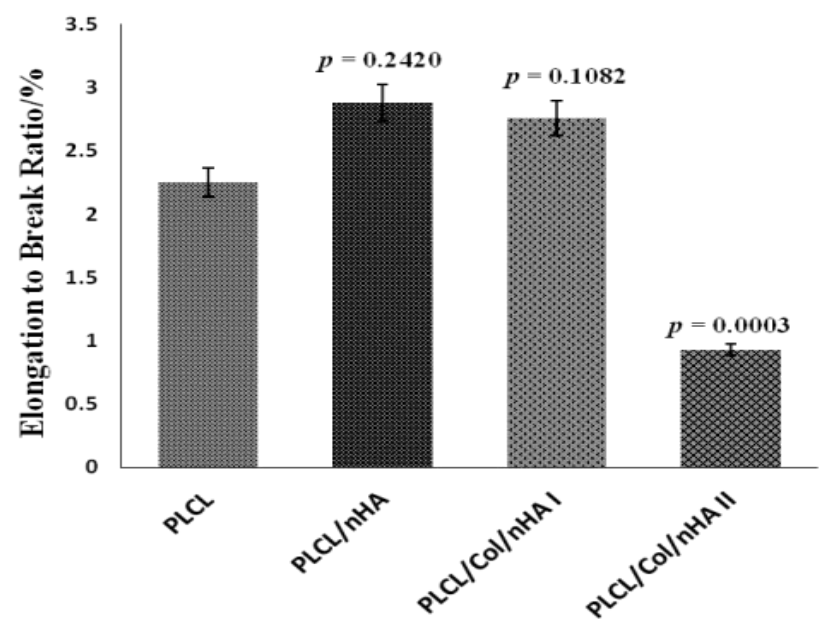

Figure 3. The anticoagulant properties of the stents assessed through recalcification time.

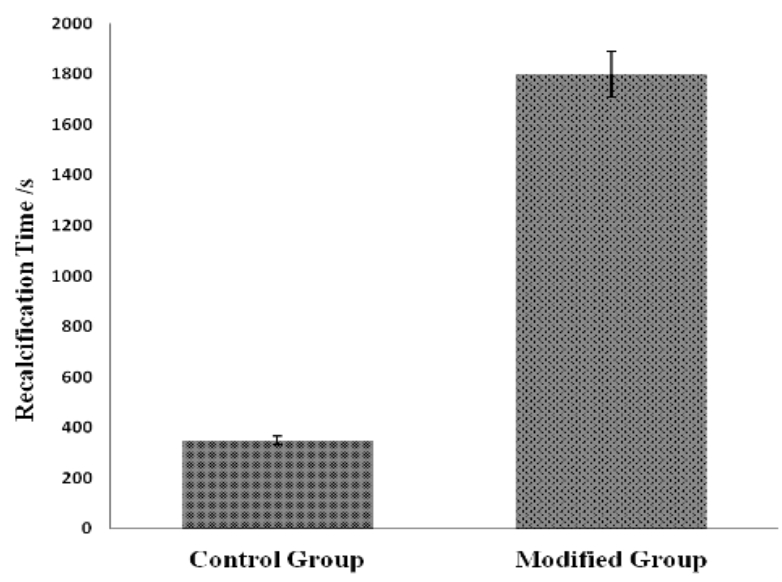




\subsection{Preparation and Characterization of New Nano-Composite Scaffolds Loaded with Vascular Stents}

The general appearance of nano-composite scaffolds is white, and porous, with a highly uniform thickness and rough texture (Figure 4A(a)). The small stents were embedded in the nano-composite scaffolds and can be viewed from the schematic diagram (Figure 4A(b) black arrows instruction), the diameter and thickness of which are about $1 \mathrm{~mm}$ and $100 \mu \mathrm{m}$.

The nano-composite scaffolds showed a porous structure, with a highly uniform pore distribution. The pores were well-connected, as could be seen after the images were magnified in Figure 4B(a,b,c). The pore size was 220 300 $\mu \mathrm{m}$. SEM images of the small stents embedded in the nano-composite scaffolds can be viewed in Figure 4B(d) (red arrows instruction).

Figure 4. The surface morphology of nano-composite scaffolds observed by naked eye (A) and SEM (B).

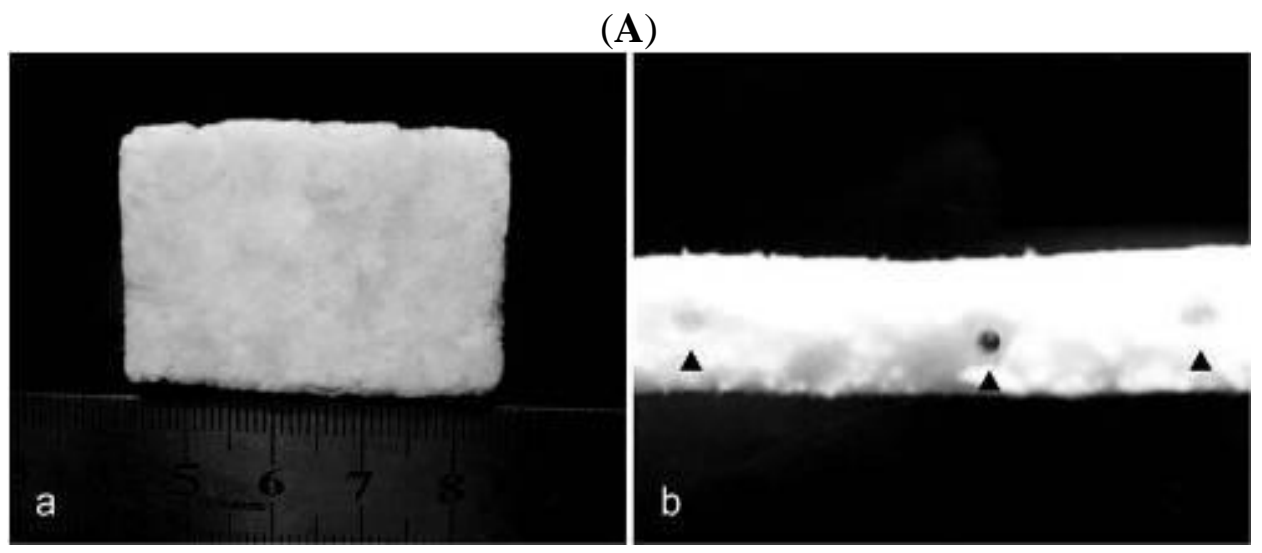

(B)
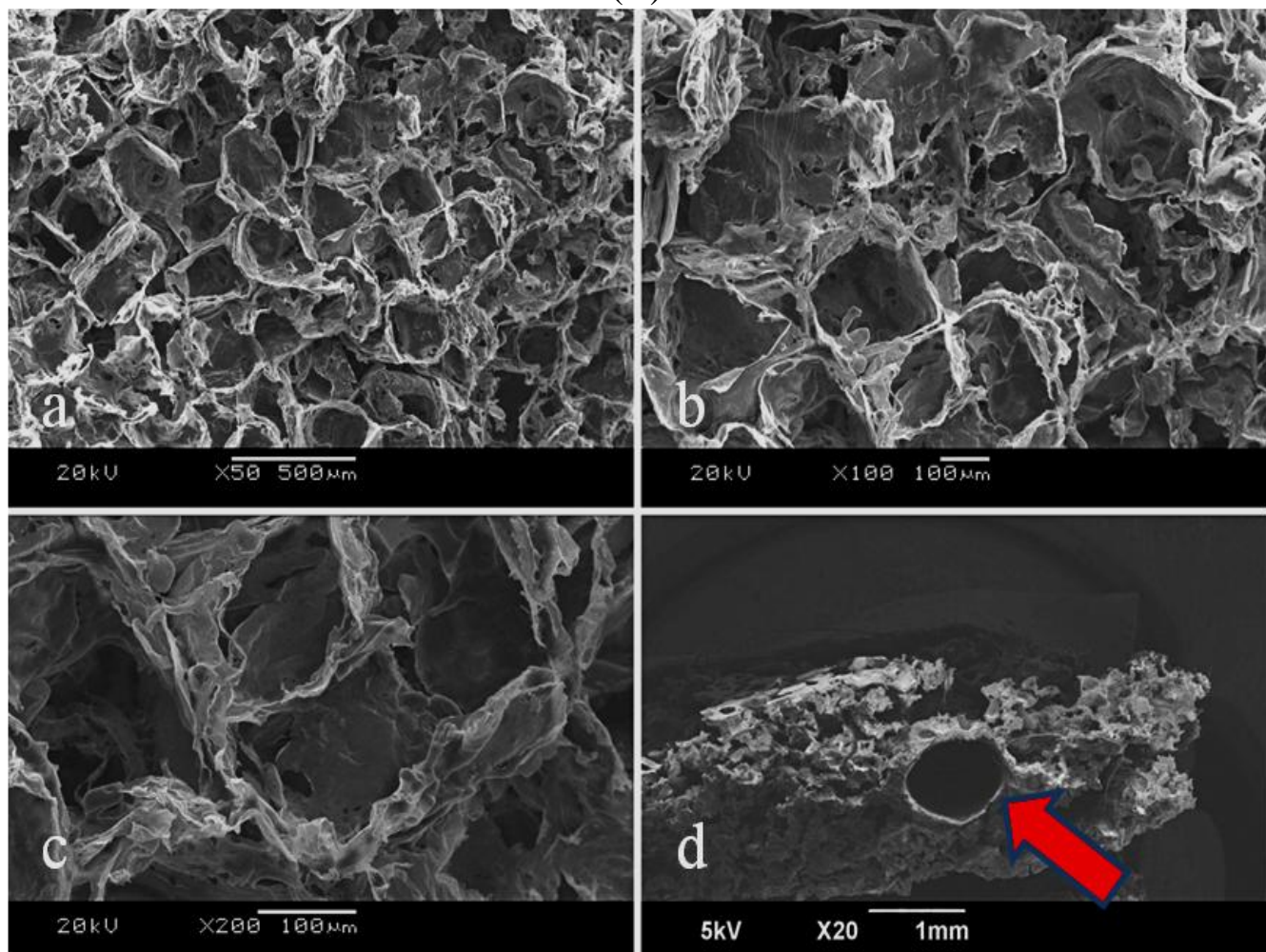
In the contact angle tests, the contact angle of PLGA/PCL/nHA group slightly increased than PLGA/PCL group (all $p$ values compared with no nHA are larger than 0.05 ) that may be due to nano-scale effect (Figure 5A). In water absorption experiments, the amount of water absorption by the nano-composite scaffolds (PLGA/PCL/nHA) exhibited rapid growth in the first $12 \mathrm{~h}$, followed by a much lower growth rate (Figure 5B).

Figure 5. Hydrophilic performance of nano-composite scaffolds assessed by contact angle tests and water absorption experiments.

(A)

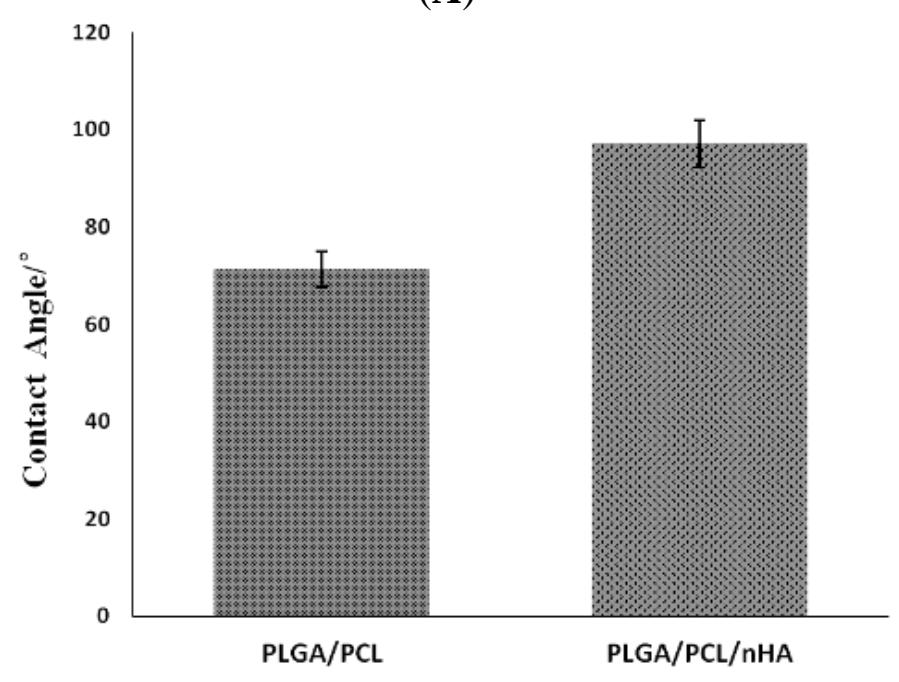

(B)

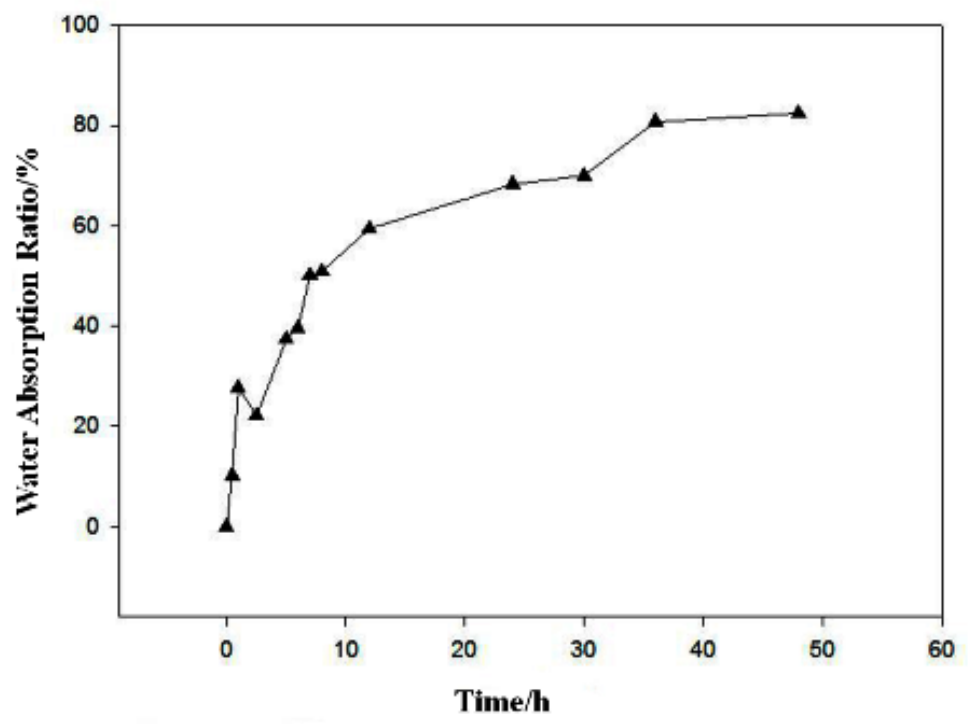

The compressive strength of nano-composite scaffolds was higher than the control group, which could be seen from Figure 6, demonstrating that strength was increased significantly through addition of nHA (all $p$ values compared with no nHA are less than 0.01 ). 
Figure 6. The compressive strength of nano-composite scaffolds.

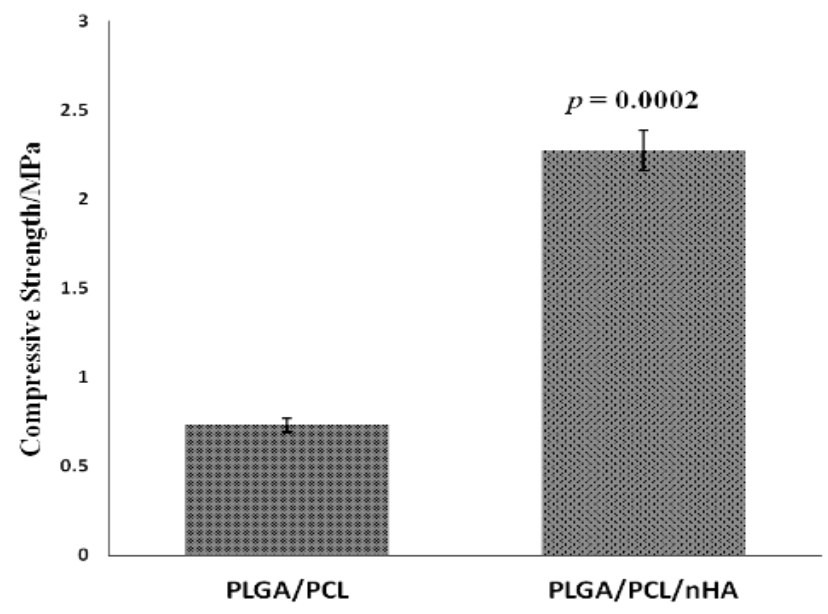

Degradation is an important factor in tissue engineering scaffolds and can impact significantly on cell growth, tissue regeneration and the host response. Therefore, the degradation rate of scaffolds should be synchronized with the cell growth rate. In this study, the $\mathrm{pH}$ of scaffolds all decreased with degradation time (Figure 7A), mainly because of the lactic acid produced during the degradation of PLGA and PCL. However, the pH of pure PLGA/PCL decreased more quickly than PLGA/PCL/nHA, perhaps as a result of the neutralization of nHA and lactic acid. Similarly, the weight losses of pure PLGA/PCL were also greater than for PLGA/PCL/nHA (Figure 7B). In addition, the early degradation rates of scaffolds were all relatively slow and accelerated rapidly after $60 \mathrm{~h}$. These results indicate that the PLGA/PCL/nHA scaffolds are more suitable for cell growth and proliferation.

Figure 7. The changes in $\mathrm{pH}$ and weight during the process of in vitro degradation of nano-composite scaffolds. A represents $\mathrm{pH}$ curves and $\mathrm{B}$ represents the weight loss curves.

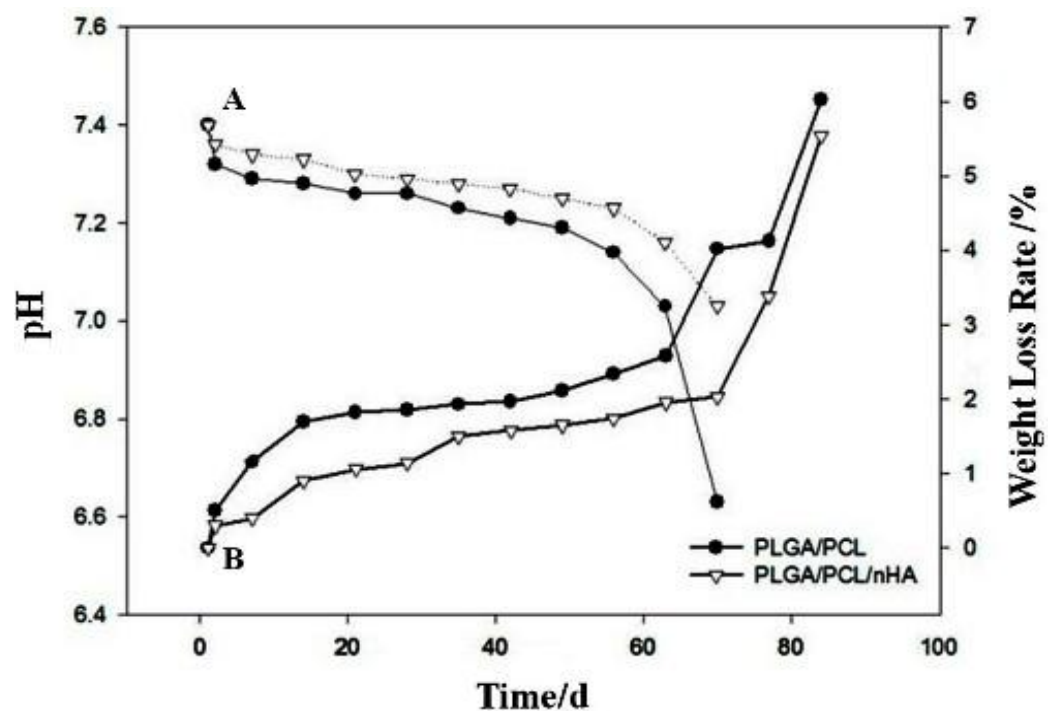

From Figure 8a,b, we could see a large number of living cells lived within the scaffolds and only a small amount of apoptosis. These results showed that the scaffolds possess good biocompatibility. 
From Figure 9a-d, it could see that scaffolds co-cultured with BMSCs after 7 days showed degradation in some areas, while cells exhibited monolayer attachment on the surface of scaffolds. Hydroxyapatite crystals appeared on the surface and inner wall of the scaffolds.

Figure 8. The cell activity of bone marrow mesenchymal stem cells (BMSCs) which seeded on scaffolds of PLGA/PCL/nHA loaded with vascular stents for 7 days was tested by DAPI and EB staining and observed through fluorescence microscopy: blue dots represent living cells (a) and dead cells were red (b).
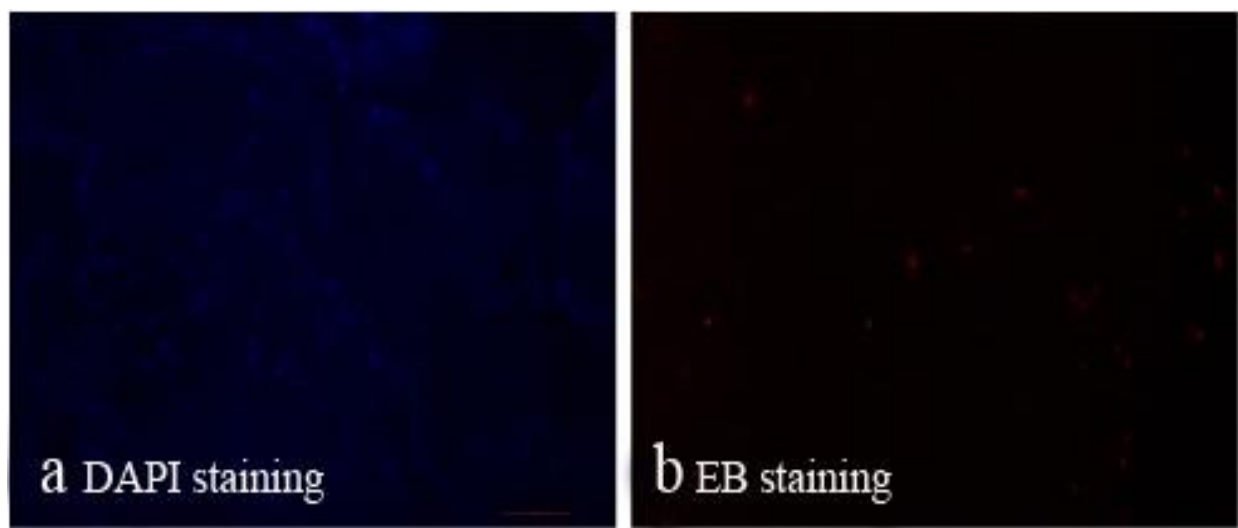

Figure 9. The changes in surface morphology of nano-composite scaffolds seeded with BMSCs after 7 days. SEM images of different magnifications: (a) $\times 50$; (b) $\times 100$; (c) $\times 200$; (d) $\times 500$.
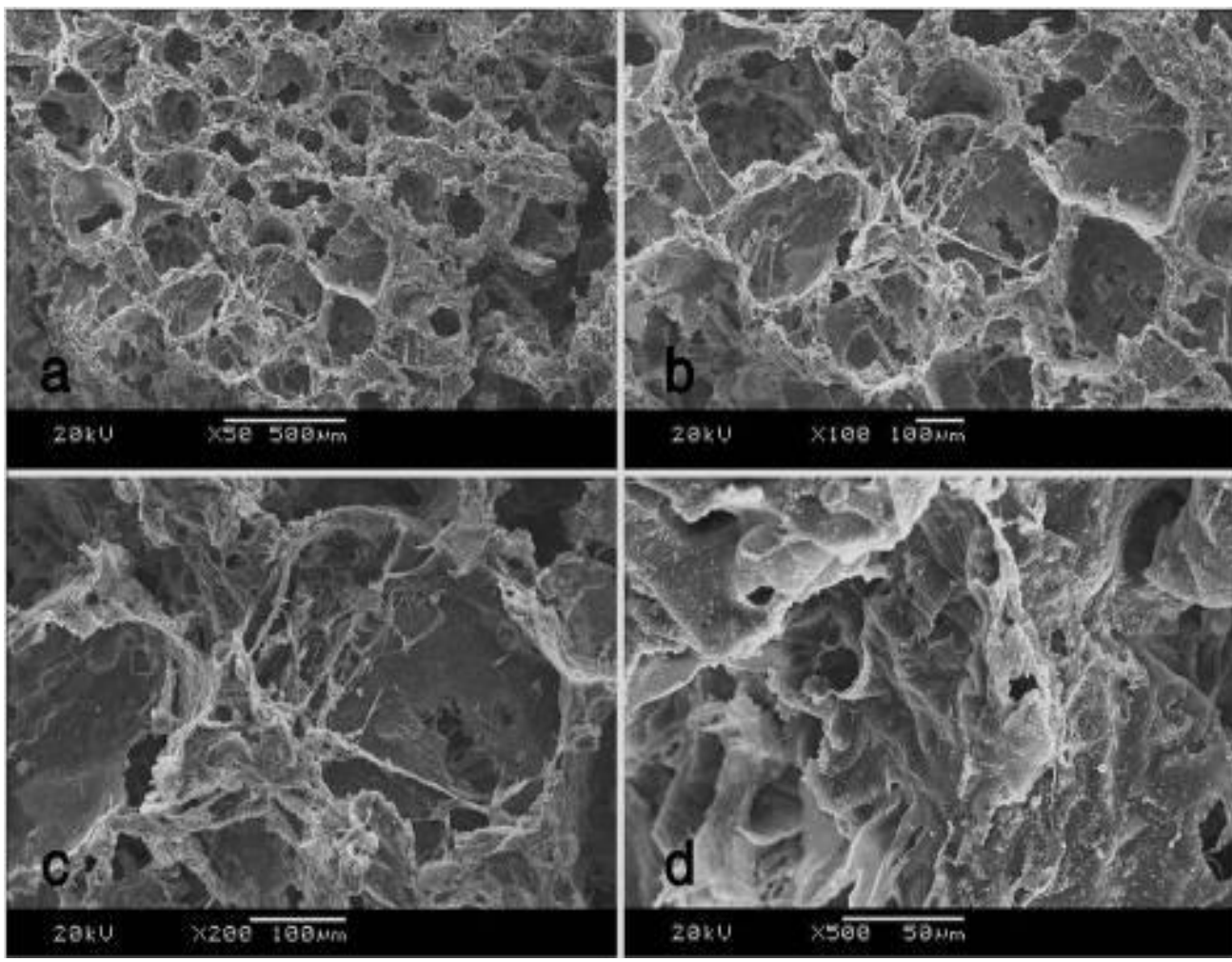

At 4 weeks after surgery, the shadow areas of low-density in bone defects of PLGA/PCL/nHA loaded with vascular stents group had little changes compared with BMSCs/PLGA/PCL/nHA loaded 
with vascular stents group, which only slightly increased in the edges of defect areas (as Figure 10 red frame instruction). After 8 weeks, increasing mineralization in the implants was observed, and group $b$ mandibles demonstrated greater new bone formation than the other group. At 12 weeks after implantation, the radio density in bone defects of $b$ group had closed to the surrounding normal bones especially in the edges of bone defects areas. Although, in the middle of the bone defects, the density was not uniform, that has demonstrated successful bone regeneration.

Figure 10. X-ray films of bone regeneration after 4, 8, 12 weeks of implanting operation. a,b represents PLGA/PCL/nHA loaded with vascular stents group and BMSCs/PLGA/PCL/ nHA loaded with vascular stents group respectively. Note: better bone formation and earlier mineralization in group $\mathbf{b}$ than group $\mathbf{a}$.

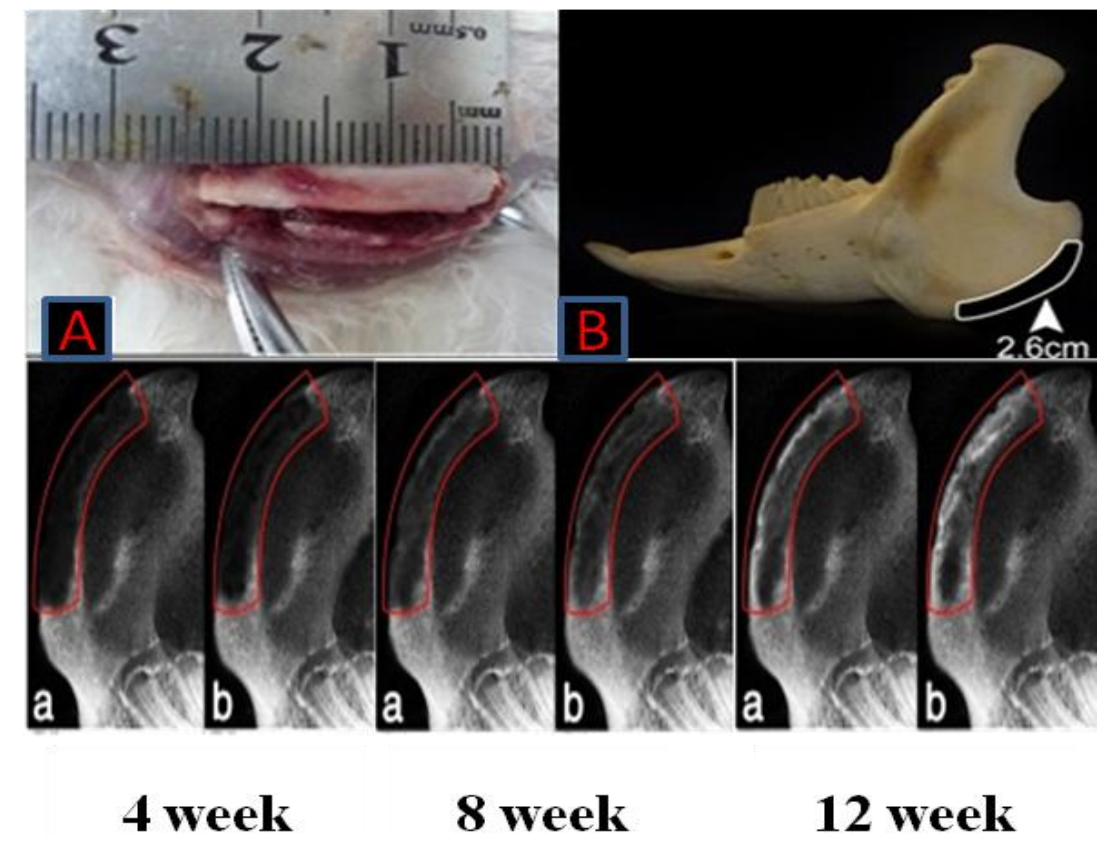

\section{Experimental Section}

\subsection{Isolation and Culture of BMSCs}

New Zealand rabbits' bone marrow mesenchymal stem cells (BMSCs) were isolated from the tibiae by bone marrow washing combined with density gradient centrifugation, suspended in Dulbecco's Modified Eagle Medium (DMEM) with 10\% fetal bovine serum (FBS) (Gibco BRL), 100 U/mL penicillin and $100 \mu \mathrm{g} / \mathrm{mL}$ streptomycin, and incubated at $37{ }^{\circ} \mathrm{C}$ with $5 \%$ humid $\mathrm{CO}_{2}$. The procedure was carried out as the method described by $\mathrm{Hu}$ et al. [32]. Cells reached $80 \%$ confluence in $8 \sim 10$ days and then were suspended for passage. Cells of passage 3 were used to seed on scaffolds and for experiments in vitro and in vivo. Media was changed every 3 days.

\subsection{Vascular Stent and Nano-Composite Scaffold Preparation}

Under room temperature, PLCL $\left(\mathrm{M}_{\mathrm{w}}, 246524\right)$ was dissolved in hexafluoro isopropyl alcohol with magnetic stirring. Collagen and nHA [33] which were produced by institute of Nano- and Bio-Polymeric Materials of School of Materials Science and Engineering in Tongji University were 
then added, with stirring to uniformity by magnetic stirrer and ultrasonication for $5 \mathrm{~min}$. The above prepared solution was injected by a $20 \mathrm{~mL}$ syringe, fixed on a syringe pump. The metal needle of the syringe was connected to the anode of a high voltage power supply, the outer diameter of which was $1.2 \mathrm{~mm}$. The receiver was a motor or foil with a rotating shaft located about $17 \mathrm{~cm}$ from the needle. The flow rate was then set to $2 \mathrm{~mL} / \mathrm{h}$, the voltage controlled to about $17 \mathrm{kV}$ and the speed of the motor at $500 \mathrm{rpm} / \mathrm{min}$. After approximately $2.5 \mathrm{~h}$ of electrospinning, the tubular stents were prepared and dried for $24 \mathrm{~h}$ under vacuum at room temperature.

In addition, PLGA and PCL (mass ratio 7:3) were dissolved in chloroform solution, with magnetic stirring for complete dissolution to a uniform polymer solution. nHA was added to the solution and dispersed evenly through ultrasonication. Sodium chloride particle sizes of 224 300 $\mu \mathrm{m}$ were obtained by a standard sieve and added to the above polymer solution, and stirred with a glass stirrer to form a uniform paste containing pore-forming agents and with a specific mobility. The paste mixture was then poured into the dish and a polymer film obtained after solvent evaporation, which was then dried in a vacuum oven and cut to appropriate sizes. The shredded polymer film and vascular stent (diameter of $0.9 \mathrm{~mm}$ ) were placed in a mold, then placed into a hot-press at $6 \sim 7 \mathrm{MPa}$ pressure at $70{ }^{\circ} \mathrm{C}$ for $3 \mathrm{~min}$. Finally, the solution was tested with silver nitrate until no white precipitate was observed, and the vascular stent support rods were removed and dried.

\subsection{Characterization}

\subsubsection{Morphology}

The morphology of the vascular stents, nano-composite scaffolds and complexes of cells and scaffolds were studied by SEM (Hitachi S-2360N). All specimens were fixed by $2.5 \%$ glutaraldehyde, dehydrated with graded ethanol, dried at the critical point and the surface coated with gold before SEM observation.

\subsubsection{Cell Activity}

The cell activity of bone marrow mesenchymal stem cells (BMSCs) which were seeded onto scaffolds of PLGA/PCL/nHA loaded with vascular stents for 7 days was tested by DAPI and EB staining. The samples of scaffolds seeded BMSCs were added $100 \mu \mathrm{L}$ DAPI and EB staining solution and incubated at $37^{\circ} \mathrm{C}$ for $15 \mathrm{~min}$. Then fluorescence microscopy was used to observe the morphology of living and dead cells at the wavelength conditions of $490 \mathrm{~nm}$ and $545 \mathrm{~nm}$ respectively. The cell activity was tested by calculating the percentage of living cells of the total cells.

\subsubsection{Hydrophilic Performance}

The hydrophilic performances of nano-composite scaffolds were measured by detection of the contact angle and water absorption ability. The samples were prepared as polymer films and contact angles were measured by the sessile drop method. In addition, specimens with dimensions $10 \times 10 \times 20 \mathrm{~mm}$ were dried and weighed as $\mathrm{W}_{1}$, then immersed and soaked in deionized water at $25{ }^{\circ} \mathrm{C}$. Subsequently, the specimens were removed from the water at specified times of 1, 2, 4, 8, 12, 24 and $48 \mathrm{~h}$, the excess 
water on the surface of the scaffold was blotted with filter paper and the samples then weighed as $\mathrm{W}_{2}$. The water absorption ratio, a, of specimens at different times is defined as:

$$
\mathrm{a}=\left(\mathrm{W}_{2}-\mathrm{W}_{1}\right) / \mathrm{W}_{1} \times 100 \%
$$

\subsubsection{Mechanical Properties}

The tensile strength of vascular stents and the compressive strength of nano-composite scaffolds were evaluated using a computer controlled tension tester (TUV CMT6104) and pressure tester (DXLL-5000). The tension tester tested the tensile strength of fiber membrane samples with dimensions $80 \times 10 \mathrm{~mm}$, which represented the vascular stents. The effective distance was $20 \mathrm{~mm}$ and the test speed was $10 \mathrm{~mm} / \mathrm{min}$. In addition, the compressive strength of round, flaky specimens with dimensions $15 \mathrm{~mm}$ (diameter) $\times 3 \mathrm{~mm}$ (thickness) was evaluated according to the national test standards of China for compression of rigid cellular plastics (GB 8813-1988). The testing was carried out under the following conditions: room temperature, dry and a loading speed of $1 \mathrm{~mm} / \mathrm{min}$. Compressive strength was calculated as follows:

$$
\sigma \mathrm{c}=\mathrm{P} / \mathrm{A}
$$

where $\sigma \mathrm{c}$ represents the compressive strength; $p$ is the load at $30 \%$ stent compression; and A is the cross-sectional area $\left(\mathrm{mm}^{2}\right)$.

\subsubsection{Anticoagulant Property}

(1) Solution configuration: Polyetherimide (PEI) solution, $1 \mathrm{mg} / \mathrm{mL}$; HOAc-NaOAc buffer solution, pH 3.8; HS and CTS solution, $1 \mathrm{mg} / \mathrm{mL}$; (2) Stent surface activation: Stents were immersed in PEI solution for $10 \mathrm{~min}$, removed, rinsed with distilled water and dried; (3) Stent anticoagulation modification: First, stents were immersed in HS solution for $20 \mathrm{~min}$, removed, washed with distilled water and dried; then they were immersed in the CTS solution for $20 \mathrm{~min}$ and the above steps repeated 10 times to modify alternately with heparin and chitosan in the outermost layer. Afterwards, fresh $\mathrm{Ca}^{2+}$-depleted plasma, $0.025 \mathrm{~mol} / \mathrm{L} \mathrm{CaCl}_{2}$ and fiber membranes were placed into an incubator at $37{ }^{\circ} \mathrm{C}$ for $10 \mathrm{~min}$ and then removed; $20 \mu \mathrm{L}$ of the plasma and $20 \mu \mathrm{L}$ of the $\mathrm{CaCl}_{2}$ solution were absorbed by pipette successively and dripped onto fiber membrane, while stirring the plasma with a small glass needle and recording the times of $\mathrm{CaCl}_{2}$ solution addition and production of filamentous fibers. The time of anti-clotting recalcification was measured by time interval.

\subsubsection{Degradability}

Specimens with a dimension of $8 \mathrm{~mm}$ (diameter) $\times 2 \mathrm{~mm}$ (thickness) were accurately weighed and placed into numbered test tubes. Then $10 \mathrm{~mL}$ of PBS buffer solution were added to the tubes and they were placed into a constant temperature bath at $37{ }^{\circ} \mathrm{C}$. The phosphate buffered saline (PBS) buffer solution was changed once a day for the first two days and then changed weekly, and the $\mathrm{pH}$ value recorded with time. Corresponding samples were removed at 1, 2, 4, 7, 14, 21, 28, 35, 42, 49, 56, 63, and 70 days, and the rate of weight loss of the scaffolds and the changes in $\mathrm{pH}$ measured with degradation time. 


\subsubsection{Preparation of the Bone Defect Model and Implantation of Experimental Material}

Thirty healthy New Zealand white rabbits weighing about $2.5 \mathrm{~kg}$ each were divided into two groups: group a $(n=15)$ : PLGA/PCL/nHA loaded with vascular stents group (served as control). Group $\mathrm{b}(n=$ 15): BMSCs/PLGA/PCL/nHA loaded with vascular stents group. The rabbits were anesthetized with pentobarbital sodium $(0.2 \mathrm{~mL} / \mathrm{kg})$. A $3 \mathrm{~cm}$ incision was made on the bilateral jaw of the rabbits. A critical size defect of $26 \times 5 \times 3 \mathrm{~mm}^{3}$ was made in a buccolingual direction on both sides of the mandible. The same size porous scaffolds or scaffold/cell constructs were inserted into the defects (Figure 10A,B). Five rabbits randomly selected from each group were sacrificed at 4, 8, and 12 weeks after implantation, respectively. Their mandibles were harvested and processed for $\mathrm{X}$-ray detection.

\subsubsection{X-ray Detection}

After sacrifice, lateral radiographs of the mandibles were taken with the mandibles positioned at $10 \mathrm{~cm}$ from the X-ray tube. The X-ray unit (Gendex DEN S-O-MAT, Milano, IL, USA) was set at $70 \mathrm{kV}$ and $7 \mathrm{~mA}$ with a $0.26 \mathrm{~s}$ exposure time.

\subsubsection{Statistical Analysis}

All data were expressed as means \pm SD (standard deviation). One-way analysis of variance (ANOVA) and Student's $t$ test were conducted to compare differences between groups using SPSS 14.0 [34]. Differences were considered to be significant when $p<0.05$ or $p<0.01$.

\section{Conclusions}

PLCL/Col/nHA vascular stents were successfully prepared by electrospinning and the surface of the stents modified by PEI, heparin and chitosan. The stents can be used in tissue engineering as small vessel stents with good mechanical, biological and anticoagulant properties. In addition, new nano-composite PLGA/PCL/nHA scaffolds loaded with the PLCL/Col/nHA vascular stents were prepared by a thermoforming-particle leaching method. These can serve as ideal scaffolds for tissue engineering with good mechanical, biological and osteogenic properties for related experimental studies of bone tissue engineering both in vivo and in vitro.

\section{Acknowledgments}

This project was sponsored by the National Science Foundation of China (NSFC-30840031, 30970726, 30800230), Nano Science and Technology Special Funding of Shanghai Science and Technology Commission (Grant Number: 0852nm03600, 11nm0504300).

\section{References}

1. Johnson, E.O.; Troupis, T.; Soucacos, P.N. Tissue-engineered vascularized bone grafts: Basic science and clinical relevance to trauma and reconstructive microsurgery. Microsurgery 2011, 31, 176-182. 
2. Lu, H.H.; El-Amin, S.F.; Scott, K.D.; Laurencin, C.T. Three-dimensional, bioactive, biodegradable, polymer-bioactive glass composite scaffolds with improved mechanical properties support collagen synthesis and mineralization of human osteoblast-like cells in vitro. J. Biomed. Mater. Res. A 2003, 64, 465-474.

3. Saranya, N.; Saravanan, S.; Moorthi, A.; Ramyakrishna, B.; Selvamurugan, N. Enhanced osteoblast adhesion on polymeric nano-scaffolds for bone tissue engineering. J. Biomed. Nanotechnol. 2011, 7, 238-244.

4. Karageorgiou, V.; Kaplan, D. Porosity of 3D biomaterial scaffolds and osteogenesis. Biomaterials 2005, 26, 5474-5491.

5. Liu, X.; Ma, P.X. Polymeric scaffolds for bone tissue engineering. Ann. Biomed. Eng. 2004, 32, 477-486.

6. Kanczler, J.M.; Oreffo, R.O. Osteogenesis and angiogenesis: the potential for engineering bone. Eur. Cell Mater. 2008, 15, 100-114.

7. L'Heureux, N.; Dusserre, N.; Konig, G.; Victor, B.; Keire, P.; Wight, T.N.; Chronos, N.A.; Kyles, A.E.; Gregory, C.R.; Hoyt, G.; et al. Human tissue-engineered blood vessels for adult arterial revascularization. Nat. Med. 2006, 12, 361-365.

8. Waksman, R.; Pakala, R. Biodegradable and bioabsorbable stents. Curr. Pharm. Des. 2010, 16, 4041-4051.

9. Ku, S.H.; Park, C.B. Human endothelial cell growth on mussel-inspired nanofiber scaffold for vascular tissue engineering. Biomaterials 2010, 31, 9431-9437.

10. Yost, M.J.; Baicu, C.F.; Stonerock, C.E.; Goodwin, R.L.; Price, R.L.; Davis, J.M.; Evans, H.; Watson, P.D.; Gore, C.M.; Sweet, J.; et al. A novel tubular scaffold for cardiovascular tissue engineering. Tissue Eng. 2004, 10, 273-284.

11. Gupta, D.; Venugopal, J.; Mitra, S.; Giri Dev, V.R.; Ramakrishna, S. Nanostructured biocomposite substrates by electrospinning and electrospraying for the mineralization of osteoblasts. Biomaterials 2009, 30, 2085-2094.

12. He, W.; Ma, Z.; Teo, W.E.; Dong, Y.X.; Robless, P.A.; Lim, T.C.; Ramakrishna, S. Tubular nanofiber scaffolds for tissue engineered small-diameter vascular grafts. J. Biomed. Mater. Res. A 2009, 90, 205-216.

13. Francis, L.; Venugopal, J.; Prabhakaran, M.P.; Thavasi, V.; Marsano, E.; Ramakrishna, S. Simultaneous electrospin-electrosprayed biocomposite nanofibrous scaffolds for bone tissue regeneration. Acta Biomater. 2010, 6, 4100-4109.

14. Du, F.; Wang, H.; Zhao, W.; Li, D.; Kong, D.; Yang, J.; Zhang, Y. Gradient nanofibrous chitosan/poly varepsilon-caprolactone scaffolds as extracellular microenvironments for vascular tissue engineering. Biomaterials 2012, 33, 762-770.

15. Zhou, M.; Liu, Z.; Wei, Z.; Liu, C.; Qiao, T.; Ran, F.; Bai, Y.; Jiang, X.; Ding, Y. Development and validation of small-diameter vascular tissue from a decellularized scaffold coated with heparin and vascular endothelial growth factor. Artif. Organs 2009, 33, 230-239.

16. Luo, L.L.; Wang, G.X.; Li, Y.L.; Yin, T.Y.; Jiang, T.; Ruan, C.G. Layer-by-layer assembly of chitosan and platelet monoclonal antibody to improve biocompatibility and release character of PLLA coated stent. J. Biomed. Mater. Res. A 2011, 97, 423-432. 
17. Sell, S.A.; McClure, M.J.; Garg, K.; Wolfe, P.S.; Bowlin, G.L. Electrospinning of collagen/biopolymers for regenerative medicine and cardiovascular tissue engineering. Adv. Drug Deliv. Rev. 2009, 61, 1007-1019.

18. Kolacna, L.; Bakesova, J.; Varga, F.; Kostakova, E.; Planka, L.; Necas, A.; Lukas, D.; Amler, E.; Pelouch, V. Biochemical and biophysical aspects of collagen nanostructure in the extracellular matrix. Physiol. Res. 2007, 56, S51-S60.

19. Villalobos, C.F.E.; Velasquillo, M.C.; Martínez, L.V.; Lecona, B.H.; Reyes, M.B.; Estrada, V.E.; Villegas, C.H.; Solís, A.L.; Espinosa, M.R.; Ibarra, P.L.C. Results of the experimental repair of osteochondral lesions in a pig model using tissue engineering. Acta Ortop. Mex. 2007, 21, 217-223.

20. Weng, Y.; Cao, Y.; Silva, C.A.; Vacanti, M.P.; Vacanti, C.A. Tissue-engineered composites of bone and cartilage for mandible condylar reconstruction. J. Oral Maxillofac. Surg. 2001, 59, 185-190.

21. Rivard, C.H.; Chaput, C.; Rhalmi, S.; Selmani, A. Bio-absorbable synthetic polyesters and tissue regeneration. A study of three-dimensional proliferation of ovine chondrocytes and osteoblasts. Ann. Chir. 1996, 50, 651-658.

22. Wang, J.; Yu, X. Preparation, characterization and in vitro analysis of novel structured nanofibrous scaffolds for bone tissue engineering. Acta Biomater. 2010, 6, 3004-3012.

23. Hiep, N.T.; Lee, B.T. Electro-spinning of PLGA/PCL blends for tissue engineering and their biocompatibility. J. Mater. Sci. Mater. Med. 2010, 21, 1969-1978.

24. Seyednejad, H.; Ghassemi, A.H.; van Nostrum, C.F.; Vermonden, T.; Hennink, W.E. Functional aliphatic polyesters for biomedical and pharmaceutical applications. J. Control. Release 2011, 152, 168-176.

25. Zhang, P.; Hong, Z.; Yu, T.; Chen, X.; Jing, X. In vivo mineralization and osteogenesis of nanocomposite scaffold of poly(lactide-co-glycolide) and hydroxyapatite surface-grafted with poly(L-lactide). Biomaterials 2009, 30, 58-70.

26. Wu, C.; Zhang, Y.; Fan, W.; Ke, X.; Hu, X.; Zhou, Y.; Xiao, Y. CaSiO microstructure modulating the in vitro and in vivo bioactivity of poly(lactide-co-glycolide) microspheres. J. Biomed. Mater. Res. A 2011, 98, 122-131.

27. Huang, Y.; Ren, J.; Ren, T.; Gu, S.; Tan, Q.; Zhang, L.; Lv, K.; Pan, K.; Jiang, X. Bone marrow stromal cells cultured on poly (lactide-co-glycolide)/nano-hydroxyapatite composites with chemical immobilization of Arg-Gly-Asp peptide and preliminary bone regeneration of mandibular defect thereof. J. Biomed. Mater. Res. A 2010, 95, 993-1003.

28. Xue, D.; Zheng, Q.; Zong, C.; Li, Q.; Li, H.; Qian, S.; Zhang, B.; Yu, L.; Pan, Z. Osteochondral repair using porous poly(lactide-co-glycolide)/nano-hydroxyapatite hybrid scaffolds with undifferentiated mesenchymal stem cells in a rat model. J. Biomed. Mater. Res. A 2010, 94, 259-270.

29. Huang, Y.X.; Ren, J.; Chen, C.; Ren, T.B.; Zhou, X.Y. Preparation and properties of poly(lactideco-glycolide) (PLGA)/nano-hydroxyapatite (NHA) scaffolds by thermally induced phase separation and rabbit MSCs culture on scaffolds. J. Biomater. Appl. 2008, 22, 409-432. 
30. Kim, S.S.; Park, M.S.; Jeon, O.; Choi, C.Y.; Kim, B.S. Poly(lactide-co-glycolide)/ hydroxyapatite composite scaffolds for bone tissue engineering. Biomaterials 2006, 27, 1399-1409.

31. Gibson, P.; Schreuder-Gibson, H.; Rivin, D. Transport properties of porous membranes based on electrospun nanofibers. Colloids Surf. A 2001, 187, 469-481.

32. Hu, J.; Qi, M.; Zou, S.; Li, J.; Luo, E. Callus formation enhanced by BMP-7 ex vivo gene therapy during distraction osteogenesis in rats. J. Orthop. Res. 2007, 25, 241-251.

33. Gu, S.Y.; Zhan, H.; Ren, J.; Zhou, X.Y. Sol-gel synthesis and characterisation of nano-sized hydroxyapatite powders and hydroxyapatite/poly(D,L-lactide-co-glycolide) composite scaffolds. Polym. Polym. Compos. 2007, 15, 137-144.

34. SPSS, version 14.0; IBM Corporation: Chicago, IL, USA, 2005.

(C) 2012 by the authors; licensee MDPI, Basel, Switzerland. This article is an open access article distributed under the terms and conditions of the Creative Commons Attribution license (http://creativecommons.org/licenses/by/3.0/). 